\title{
Van wyk Grumbach and café au lait: An unusual association
}

\author{
Kochar IS and Ramachandran * $^{*}$ \\ Pediatric and Adolescent Endocrinology, Indraprashta Apollo Hospital, New Delhi, India
}

\begin{abstract}
Primary hypothyroidism can present with a myriad of symptoms and needs a high index of suspicion to diagnose and treat the rare presentations of it. We report the youngest case from India of Van Wyk Grumbach due to primary hypothyroidism presenting with precocious puberty and a rare association with café au lait spots. There needs to be awareness about this condition in pediatricians and surgeons to avoid unnecessary invasive diagnostic tests.
\end{abstract}

\section{Introduction}

Primary hypothyroidism has been most commonly associated with delayed puberty, however an uncommon association with precocious puberty is seen in the presentation of Van Wyk Grumbach syndrome (VWBS) [1]. Van Wyk and Grumbach first described this in 1960 with the cardinal features of delayed bone age, juvenile hypothyroidism and isosexual precocious puberty [2]. It is one the rare causes of precocious puberty with delayed bone age and needs to be borne in mind to prevent unnecessary investigations and sometimes surgical misadventure due to suspected gonadotropin secreting tumors.

Streaked hyperpigmentation may be rarely associated with VWBS but an association with café au lait spots has not been previously reported.

\section{Case report}

A 3 year 11-month old girl child came with the complaints of vaginal bleeding of 2 episodes. She had the first episode 1 month prior, she had few episodes of spotting lasting 2-3 days. After this the child had a similar episode 1 month after the first episode, this time she had a few drops of bleeding lasting 2-3 days again.

Following the first episode the parents took the child to some local practitioner who advised an ultrasonography of the abdomen, which reported bilateral bulky multicystic ovaries suspecting a dermoid cyst. As a follow through on this report the surgeon did a diagnostic laparoscopy and sent biopsies from both the ovaries, suspecting $\mathrm{LH}$ secreting tumors. The biopsy revealed no abnormal or malignant cells, reported benign cystic lesions, histomorphological findings of normal stroma with few scattered follicles. The parents came to us for a second opinion. She was a full-term child born at term with uneventful antenatal and perinatal history; there was no history any maternal illness.

On examination she weighed $12 \mathrm{~kg}$, height was $89 \mathrm{~cm}$, mid parental height $151 \mathrm{~cm}$, both height and weight were below -1 SD. She had breast budding Tanner stage II, no axillary or pubic hair. The child also had very coarse skin, but no previous history of any chronic illness, bleeding disorders in the family, no history of trauma or sexual abuse. The neonatal period was uneventful.
She had one café-au lait spot measuring $2 \times 3 \mathrm{~cm}$ on the chest since birth, with soft margins. This was present since birth and there had been no change in the size since. With these findings we ordered a complete blood counts, PT/aPTT, LH, FSH, estradiol, anti-TPO, cortisol, LDH, HCG, thyroid function tests. TSH- >100 uIU/mL, Free T4- 1.42ng/dl, Free T3- $5.21 \mathrm{pg} / \mathrm{ml}$, hemoglobin- $10.8 \mathrm{gm}$, platelets- $3.08 \mathrm{lac} / \mathrm{cumm}, \mathrm{PT} /$ aPTT was in normal range, LH- $0.563 \mathrm{IU} / \mathrm{L}, \mathrm{FSH} 1.79 \mathrm{IU} / \mathrm{ml}$, estradiol $69.8 \mathrm{pg} / \mathrm{ml}$, anti-TPO- 860 units/ml, LDH-217, HCG $<2.00$.

Ultrasound of the thyroid revealed both lobes of the glands of normal size and homogenous echogenicity, measuring $0.9 \times 0.7 \times 1.5 \mathrm{~cm}$ and left lobe $0.8 \times 0.7 \times 1.4 \mathrm{~cm}$. A diagnosis of primary hypothyroidism was with a possibility of Van Wyk Grumbach syndrome, also a second possibility of Mc Cune Albright was kept in view of café au-lait and precocious puberty. There was no history of any thyroid disorders in the family. Her bone age was 3.3yrs and no other skeletal anomaly noted.

The child was immediately started on tablet thyroxine 50ug orally. A dynamic MRI of the sella turcica was advised which showed convexity of the superior margins of the anterior pituitary gland. No definite focal areas of differentiation were noted. The pituitary gland measured $10 \mathrm{~mm}$ in AP diameter, $11 \mathrm{~mm}$ height, $16 \mathrm{~mm}$ transverse dimensions. The pituitary stalk had normal morphology and dimensions.

The height of the pituitary was increased for the age. In view of breast budding, vaginal bleeding, multicystic ovaries, autoimmune hypothyroidism and increased pituitary height a final diagnosis of Van Wyk Grumbach was made. The association of VWGS and café aulait spots have never been reported prior in literature and can cause diagnostic dilemma if the treating physician is not aware about the syndrome.

${ }^{\star}$ Correspondence to: Smita Ramachandran, Fellow Pediatric and Adolescent Endocrinology, Indraprastha Apollo Hospital, New Delhi, India; Tel: 9818488818; E-mail-smita_rama25@yahoo.com

Key words: Van Wyk Grumbach, café au lait, precocious puberty

Received: September 14, 2018; Accepted: September 24, 2018; Published: September 28, 2018 


\section{Discussion}

VBGS has been postulated to be a consequence of hormonal overlap between TSH and FSH leading to GnRH independent puberty. It can occur in both males and females, however has been reported more frequently in girls. They present with early breast development, early onset menstrual bleeding, delayed bone age and multicystic ovaries but with lack of axillary and pubic hair [3]. There is a dose dependent stimulation of the FSH receptors by the high levels of the a subunits of $\mathrm{TSH}$, resulting in high values of estrogen causing menstrual bleeding, breast development and multicystic ovaries $[4,5]$.

Primary hypothyroidism is the central factor for the paradoxical presentation in VWGS. It is most commonly autoimmune [6,7], but congenital and acquired causes have also been reported [8]. Sella turcica enlargement is noted in long standing cases of hypothyroidism due to the thyrotroph hyperplasia, as also seen in our case. VWGS is a condition that responds extremely well to thyroxine and results in results in resolution of all the symptoms over a period of 6-12 months [1]. However clinical dilemma due to multicystic ovaries and elevate CA-125, which occurs due to delayed clearance secondary to hypothyroidism can result in unwarranted surgical interventions.

An uncommon feature seen in VWGS is streaky hyperpigmentation seen over the body which usually develops over time. It has been hypothesized to be due the melanocyte stimulating hormone (MSH) action on the skin, as a due to cross reaction between TSH and FSH $[9,10]$. Though café au-lait spots in VWGS has never been reported prior. The occurrence whether incidental or not cannot be ascertained at present. The presence of café au lait spots can however pose a dilemma differentiating from VWGS from Mc Cune Albright, as both the conditions present with early puberty and café au lait is seen commonly in the latter. A delayed bone age, hypothyroidism and absence of fibrous dysplasia differentiates the two.

\section{Conclusion}

VWGS is a rare but completely treatable condition due to long standing hypothyroidism. Despite the myriad of seemingly grave symptoms, they revert back on treatment with thyroxine. Both the physicians and surgeons need to be aware of this condition secondary to hypothyroidism to avoid unnecessary investigations and unwarranted surgical interventions.

\section{References}

1. Hachicha M, Maaloul I, Aissa K, Kamoun T, Aloulou H (2018) Van Wyk Grumbach syndrome : A rare cause of precocious puberty. Presse Med 47:483-486. [Crossref]

2. Van Wyk JJ, Grumbach MM (1960) Syndrome of precocious menstruation and galactorrhea in juvenile hypothyroidism: An example of hormonal overlap in pituitary feedback. J Pediatrics 57:416-435.

3. Panico A, Lupoli GA, Fonderico F, Colarusso S, Marciello F, et al. (2007) Multiple ovarian cysts in a young girl with severe hypothyroidism. Thyroid 17:1289-1293.

4. Anasti J, Flack M, Froehlich J, Nelson L, Nisula B (1995) A potential novel mechanism for precocious puberty in juvenile hypothyroidism. J Clin Endocrinol Metab 80:276279. [Crossref]

5. Baranowski E, Högler W (2012) An unusual presentation of acquired hypothyroidism the Van Wyk-Grumbach syndrome. Eur J Endocrinol 166: 537-542. [Crossref]

6. Sharma D, Dayal D, Gupta A, Saxena A (2011) Premature menarche associated with primary hypothyroidism in a 5.5-year-old girl. Case Rep Endocrinol 2011: 678305. [Crossref]

7. Trans S, Kim E, Chin A (2013) Severe menorrhagia, unilateral ovarian mass, elevated inhibin level and severe hypothyroidism: an unusual presentation of Van Wyk Grumbach syndrome. J Pediatr Surg 48: E51-4.

8. Chattopadhyay A, Kumar V, Karnataka M (2003) Polycystic ovaries, precocious puberty and acquired hypothyroidism: the Van Wyk and Grumbach syndrome. $J$ Pediatr Surg 38:1390-1392.

9. Motamed F, Eftekhari K, Kiani MA, Rabbani A (2012) Ovarian cyst enlargement in a 14 year old female with persistent ascities. Severe hypothyroidism and elevated serum CA-125 level Iran Red Crescent Med J 14:379-381. [Crossref]

10. Baranowski E, Högler W (2012) An unusual presentation of acquired hypothyroidism: The Van Wyk-Grumbach syndrome. Eur J Endocrinol. 166:537-542. [Crossref]

Copyright: (C2018 Kochar IS. This is an open-access article distributed under the terms of the Creative Commons Attribution License, which permits unrestricted use, distribution, and reproduction in any medium, provided the original author and source are credited. 